“(C) 2018 IEEE. Personal use of this material is permitted. Permission from IEEE must be obtained for all other uses, in any current or future media, including reprinting/republishing this material for advertising or promotional purposes, creating new collective works, for resale or redistribution to servers or lists, or reuse of any copyrighted component of this work in other works." 


\title{
EFFECT OF COMPRESSION GARMENTS ON CARDIOVASCULAR FUNCTION DURING RECOVERY PHASE
}

\author{
Thi Nhu Lan Nguyen, Student Member, IEEE, David Eager, Member, IEEE, Hung Tan Nguyen, \\ Senior Member, IEEE
}

\begin{abstract}
The aim of this present research was to determine whether the cardiovascular function has been affected by wearing compression garments during the recovery phase. Fourteen subjects (men, $n=7$; women, $n=7 ; 24.7 \pm 4.5$ years, $166.0 \pm 7.6 \mathrm{~cm} ; 60.9 \pm 12.0 \mathrm{~kg}$ ) completed a running protocol on a treadmill. Each subject participated in two running experiments, using either compression garments (CGs) or noncompression garments (NCGs) during exercise and 2 hours recovering time. Electrocardiogram (ECG) signals were collected during 2 hours recovery using wearable sensors. The present work indicated a statistically significant difference between CGs and NCGs from 90 minutes recovery onwards $(p<0.05)$. ECG parameters showed some significant difference in heart rate $(\mathrm{HR}), \mathrm{ST}$ and corrected QT $(\mathrm{QTc})(\mathrm{p}<0.05)$. Therefore, the cardiovascular function was positively influenced by the application of CGs during the recovery phase.
\end{abstract}

\section{INTRODUCTION}

Restoration of performance for athletes is essential to maximize competitive success [1]. Nutrition, massage and training methods were investigated as possible strategies [2, 3]. Specifically, many previous studies recommended CGs as a recovery modality after exercise and competition [4, 5]. Compression group demonstrated many effects on wearers on recovery such as substantially lower values in delayed onset muscle soreness when compared with the placebo group during recovery $[4,6]$.

Similarly, the utilization of compression clothing reduced lactate dehydrogenase, perceptual measurements, and muscle swelling [7, 8]. A study of the relationship between CGs and recovery suggested that the repair processes and alteration in the inflammatory response to damage were accelerated an inside the muscles by applying of CGs [9]. Although many scientific articles suggested that the use of CGs produces positive effects post exercise, some others also reported that the rate of recovery following activity or the extent of peripheral fatigue was not affected by the application of CGs [10]. Therefore, the effect of CGs on recovery phase has not been clearly established.

Thi Nhu Lan Nguyen, David Eager are with the Faculty of Engineering and Information Technology at University of Technology Sydney, New South Waves 2007, Australia, (email: ThiNhuLan.Nguyen@student.uts.edu.au, David.Eager@uts.edu.au).

Hung Nguyen is with the Faculty of Science, Engineering and Technology at Swinburne University of Technology, Victoria 3122 Australia, (email: hungnguyen@swin.edu.au).
Evaluation of RR intervals calculated heart rate variability (HRV) by ECG signals [11] which were associated with the state the autonomic nervous system [12]. Evidence as to whether CGs has affected HRV can illustrate valuable information relating to autonomic control of the cardiovascular system. Additionally, HR parameters after exercise also have a significant role in monitoring modifications in performance and examine as a useful method of the training process in both athletes and nonathletes [13]. Particularly, many research examined the relationship between slow HRR and carotid atherosclerosis. For instance, slow HRR increased the risk of endothelial dysfunction, autonomic dysfunction, metabolic syndrome, cardiovascular mortality and diabetes [14].

Consistent with that, the development of hypertension was also associated with higher values of HR [15]. The risk of increasing hypertension can also be predicted by a higher level of resting HR [16]. Similarly, lower values of NN50 would be a sign of more significant mental stress [17]. However, there is still currently little understanding regarding HRV dynamics mechanisms underlying the effects of CGs on runners during recovering [18].

ECG signals also provide other additional parameters which include TpTe, QRS, ST, QT and corrected of QT (QTc) rather than only HRV. Some previous observations represented the relationship between prolongation of QT intervals or QTc and the development in cardiac arrhythmias [19]. The longer values of QTc dispersion and QT dispersion and QT dispersion has also affected the performance of athletes in training [20]. In the same vein, MC Mandyam 2012 indicated that longer QT interval was associated with an increase in stroke and incident atrial fibrillation [21]. At present, no previous observation has represented the relationship between ECG signals and the application of CGs post exercise to the best of our knowledge.

This current research examines the underlying mechanism of cardiovascular function wearing CGs during the recovery phase by analyzing ECG signals.

\section{METHOD}

\section{A. Data collection}

Fourteen healthy participants $(24.7 \pm 4.5$ years, BMI-body max index: $21.9 \pm 3.1 \mathrm{~kg} \cdot \mathrm{m}^{-2}$, weight: $60.9 \pm 12.0 \mathrm{~kg}$, height: $166.0 \pm 7.6 \mathrm{~cm})$ conducted in the protocol. All subjects were non-athletes and do not have a smoking history. Exclusion criteria include cardiovascular, cerebrovascular and respiratory disease. Alcohol and caffeine were not accepted about 24 hours prior to the experiments. They also were required to have a healthy night sleep. All volunteers 
completed consent forms and a basic medical questionnaire before the test. The protocol was approved by Human Ethics Committee at the University of Technology Sydney. (UTS HREC REF NO. ETH16-0696). The detailed characteristics of participants (seven men and seven women) are shown in Table 1.

TABLE I. SUBJECTS CHARACTERISTICS

\begin{tabular}{|l|l|l|}
\hline \multirow{2}{*}{\multicolumn{1}{|c|}{ Parameters }} & \multicolumn{2}{c|}{ Subjects (n=14) } \\
\cline { 2 - 3 } & $\begin{array}{l}\text { Men (n=7) } \\
\text { Mean } \pm \text { std }\end{array}$ & $\begin{array}{l}\text { Women (n=7) } \\
\text { Mean } \pm \text { std }\end{array}$ \\
\hline Age (year) & $25.8 \pm 4.9$ & $23.7 \pm 40$ \\
\hline Height $(\mathrm{cm})$ & $171.6 \pm 5.0$ & $160.4 \pm 5.1$ \\
\hline Weight $(\mathrm{kg})$ & $70.7 \pm 6.6$ & $51.1 \pm 6.8$ \\
\hline Body mass index (kg.m-2) & $24.1 \pm 2.6$ & $19.8 \pm 1.9$ \\
\hline
\end{tabular}

The CGs used in the current experiment was SportSkins Classic whole body compression garments (WBCGs), from Skins, Campbelltown, NSW, Australia. The whole body CGs included long-leg pants (from waist to ankle) and a full sleeved top (from neck to waist) which comprised of $24 \%$ Roica Spandex and 76\% Nylon and Meryl Microfiber. Each CGs was fitted with size-correct garments according to the manufacturer's advice, based on subjects' body mass and stature. During the experiments, NCGs was a T-shirt and short with $0 \%$ level of pressure. In both tests, participants were required to wear the same socks and shoes. The kind of comparison garments is shown in Figure 1.

Each participant visited the laboratory twice throughout the trials period on at least two separate days. CGs and NCGs were randomized during the two running tests. The normal laboratory temperature was set at a range of $20-22^{\circ} \mathrm{C}$ in the both tests.

Subjects participated in two running tests with CGs and without CGs on two different days. A rest of about 10 minutes before the tests was required. ECG electrodes were attached to the lead II position (Figure 1). Data of ECG-rest were collected after this step.

Figure 1. Subject wear compression garments (a1) and non-compression garments (a2). Lead II position (b). Detection of intervals (c)

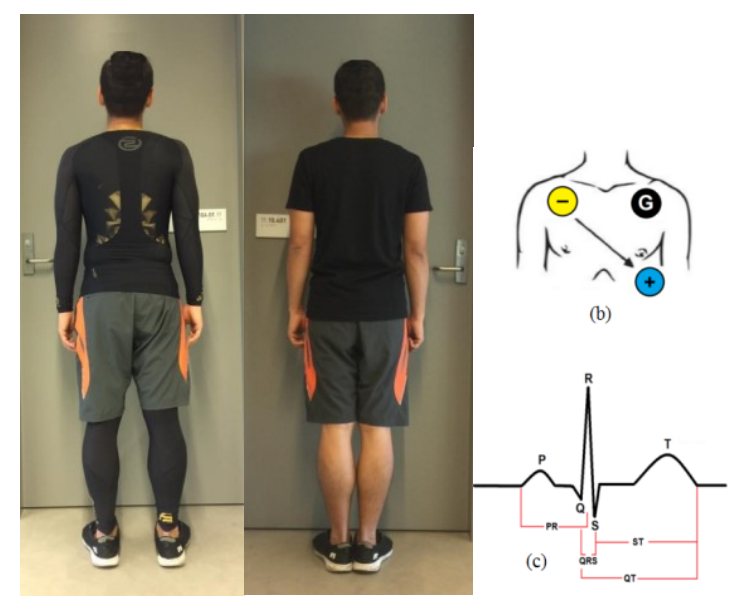

(a1)

(a2)
Participants completed a 10-minute running on a treadmill at $6 \mathrm{~km} / \mathrm{h}$ on $0 \%$ grade. After finishing every $2-$ minute running, the velocity of treadmill then was increased by $1 \mathrm{~km} / \mathrm{h}$. The process continued until a speed of $11 \mathrm{~km} / \mathrm{h}$ was reached. ECG signals were collected for 2 hours after the exercise using a Flexcomp Infiniti Monitor of Thought Technology Ltd, Canada.

\section{B. Data analysis}

All analyses were calculated with Matlab version 2016 b. The mean value (mean) and standard deviation (std) were computed for all descriptive and physiological measures. Statistical significance is reported when a p-value is lower than 0.05 .

Heart rate variability (HRV) comprises HR, RMSSD, SDNN, pNN50, NN50, SD1, SD2, SD1/SD2, HF (from 0.15 to $0.4 \mathrm{~Hz}$ ), LF (from 0.04 to $0.15 \mathrm{~Hz}$ ), VLF (from 0.003 to $0.04 \mathrm{~Hz}$ ), and the ratio of $\mathrm{LF} / \mathrm{HF}$. These parameters are delineated by an assessment of RR interval variability. ECG intervals such as QRS, QT, ST, and TpTe were calculated from Q, S, Tp, Te intervals throughout 20 seconds after each 10-min recovery. The corrected QT intervals (QTc) were estimated based on Bazett's formula as shown below.

$$
Q T c=\frac{Q T}{\sqrt{R R}}
$$

\section{RESULTS}

The application of compression garments produced substantially lower heart rate compared with non-using CGs after 30-min recovery (CGs: 92.06, NCGs: 96.04, p=0.0485). There were significant effects by the application of CGs at the end of the recovery phase, from 80 min-recovery onwards. For example, the sample was found to have significant difference at $80-\mathrm{min}$ recovery (CGs: 80.13, NCGs: $85.35, \mathrm{p}=0.0376$ ), at 90 -min recovery (CGs: 79.49 , NCGs: $85.17, \mathrm{p}=0.0066$ ), at $100-\mathrm{min}$ recovery (CGs: 79.05 , NCGs: 83.1, $\mathrm{p}=0.0072$ ), at 110-min recovery (CGs: 76.87, NCGs: 82.77, $\mathrm{p}=0.001$ ) and at 120 -min recovery (CGs: 76.39, NCGs: 82.28, $\mathrm{p}=0.0001)$. The results are shown in Figure 2.

The analysis demonstrated a significant difference with lower corrected QT in wearing CGs group with $\mathrm{p}<0.05$ and at 90-min recovery (CGs: $391.28 \mathrm{~ms}$, NCGs: $403.77 \mathrm{~ms}$, $\mathrm{p}=0.0035$ ). At 100-min recovery, there was also affected by the application of CGs with $\mathrm{p}=0.0369$ (CGs: $390.97 \mathrm{~ms}$, NCGs: $405.30 \mathrm{~ms}$ ). Similarly, QTc was influenced by using of CGs at 110-min recovery (CGs: $388.85 \mathrm{~ms}$, NCGs: 399.14 $\mathrm{ms}, \mathrm{p}=0.0073)$. At 120 -min recovery, the difference was significant again (CGs: $387.63 \mathrm{~ms}$, NCGs: $398.26 \mathrm{~ms}$, $\mathrm{p}=0.0128$ ). Comparison between using CGs and NCGs is shown in Figure 3.

ST intervals showed the significant difference after 60min recovery with $\mathrm{p}=0.0269$ (CGs: 282.64 ms, NCGs: 274.27 ms). Similarly, at 90-min recovery, CGs group demonstrated the considerable difference compared with NCGs in $\mathrm{p}=0.0452$ with $289.21 \mathrm{~ms}$ and $279.43 \mathrm{~ms}$, respectively. At 
Figure 2. Comparison between CGs and NCGs in HR $\left({ }^{*} \mathrm{p}<0.05\right)$

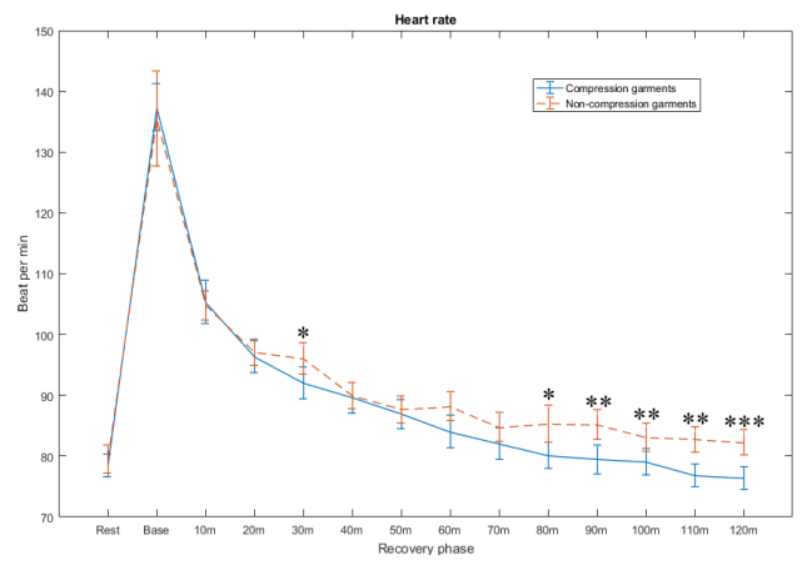

Figure 3. Comparison between CGs in NCGs in QTc $\left({ }^{*} \mathrm{p}<0.05\right)$

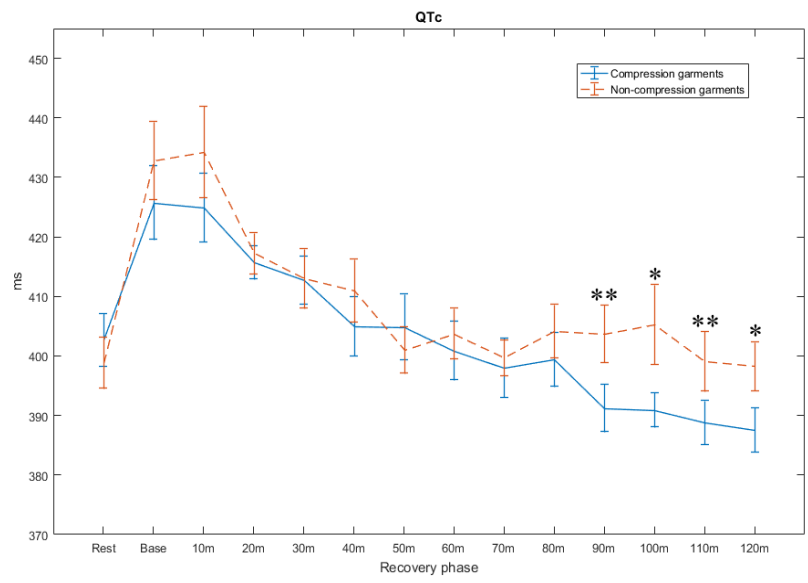

Figure 4. Comparison between CGs and NCGs in ST $\left({ }^{*} \mathrm{p}<0.05\right)$

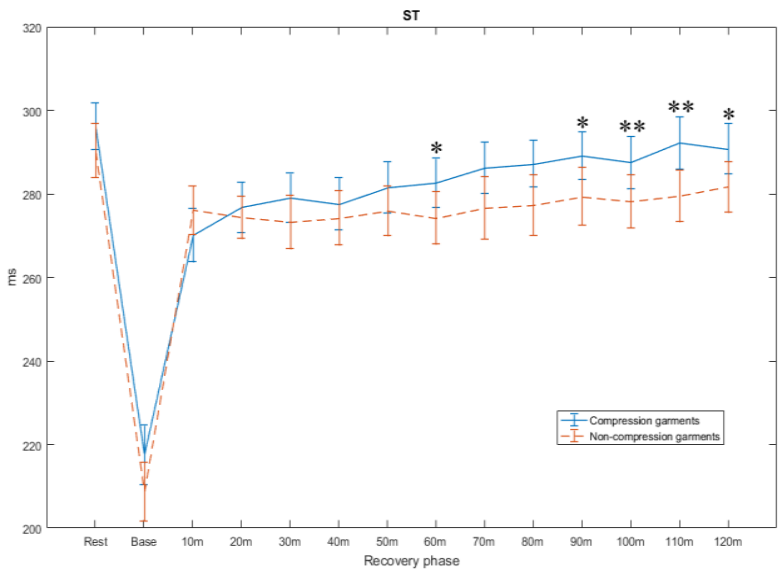

$100 \mathrm{~min}$ and $110 \mathrm{~min}$ of the recovery phase, there were p<0.001 with CGs: $287.51 \mathrm{~ms}$, NCGs: $278.2(\mathrm{p}=0.0008)$ and CGs: $292.22 \mathrm{~ms}$, NCGs: $279.47 \mathrm{~ms}(\mathrm{p}=0.0005)$, respectively. At the end of the recovery-120 min, ST also indicated a significant difference between CGs: $290.8 \mathrm{~ms}$ and NCGs: $281.68 \mathrm{~ms}$ with $\mathrm{p}=0.0216$. The results are presented in Figure 4.

\section{DISCUSSION AND CONCLUSION}

Both CGs and NCGs showed the decrease in HR during the recovery phase. During the first 80 -min recovery of the running test, there were nearly nonsignificant results between using whole body CGs and NCGs. This finding is consistent with other studies of CGs which showed no alternation in HR compared with NCGs after 20-min recovery of 6 steps incremental test on kayak ergometer [22] or 20-min recovery of 3 intermittent maximal anaerobic running power tests [23]. Similarly, studies of a match simulation exercise [7] and $40 \mathrm{~km}$ cycling time trial performance [24] indicated a nonsignificant difference in HR after haft-hour recovery using lower body (full-led length) CGs compared with NCGs. Moreover, the application of compression stocking also claimed nonsignificant difference with NCGs in a maximal cycling ergometer test after 60-min recovery [25]. However, a quicker attenuation in HR was significant in the subjects with CGs compared with NCGs after $80 \mathrm{~min}$ of recovering phase $(\mathrm{p}<0.05)$. There was an enhancement blood flow, and redistribution of blood flows as evidenced by a lower mean heart rate [26]. Moreover, high-intensity exercise can cause glycogen depletion. That led to inadequate muscle glycogen synthesis. However, the improvement of blood flow by using CGs can solve that issue [24]. The rapid recovery of heart rate in CGs group after 80-min recovery supports some previous research relating to the positive influence of CGs as a recovery strategy $[4,5]$. The finding of the current study is that the application of CGs after the running exercise may have advantageous effects on HR.

This present investigation has shown that wearing CG results in a significant lower QTc after 90-min recovery compared with wearing NCGs $(\mathrm{p}<0.05)$. Consistent with this, earlier research has demonstrated shorter corrected QT interval in a group of using CGs at the end of a running test [27]. The longer QTc is associated with an increased incidence of stroke and atrial fibrillation [21]. Moreover, some previous research indicated a significant development in the risk of cardiac arrhythmias has a relationship with the QTc prolongation [20]. This present finding also is consistent with our previous study concluding that the pressure of CGs may influence on QTc value [28]. As a result, CGs may have positive effects on recovery based on the lower QTc.

The results show that ST interval decreased at the end of the exercise and increased to the normal values during the recovery in this present study. CG group reached a longer ST interval during the recovery phase. There were significant longer ST intervals in $\mathrm{CG}$ compared to $\mathrm{NCG}$ group at 60-min recovery and after 90-min recovery onwards $(\mathrm{p}<0.05)$. Essentially, ST intervals in CG group reached their normal values quicker than the $\mathrm{NCG}$ group. Moreover, a previous observation claimed that ST intervals were significantly associated with low DBP and high SBP [29]. The result of the present study supports previous considerations relating to the effects of CGs also showed 
significant higher SBP values during the supine and standing phases [30].

Wearing whole body compression garments may assist in the recovery process, due to the effects on cardiovascular function. The observed results have positive effects on heart rate, ST, and QTc. Although further studies are required to indicate the more underlying mechanisms by which this recovery strategy is efficient, compression garments should be recommended to coaches and athletes looking to improve recovery between exercise episodes. The above findings can improve the quality of training and could incur improvement in subsequent performance.

\section{REFERENCES}

[1] F. Neferu, "The Importance Of Exercise Capacity Recovery In Obtaining Performance In Sports/Importanta Refacerii Capacitatii De Efort În Obtinerea Performantei În Sport," Studia Universitatis" Vasile Goldis". Seria Educatie Fizica si Kinetoterapie, vol. 4, no. 2, p. 75, 2015.

[2] F. E. Rossi, A. Landreth, S. Beam, T. Jones, L. Norton, and J. M. Cholewa, "The Effects of a Sports Nutrition Education Intervention on Nutritional Status, Sport Nutrition Knowledge, Body Composition, and Performance during Off Season Training in NCAA Division I Baseball Players," Journal of sports science \& medicine, vol. 16, no. 1, p. 60, 2017.

[3] C. Haas, T. A. Butterfield, Y. Zhao, X. Zhang, D. Jarjoura, and T. M. Best, "Dose-dependency of massage-like compressive loading on recovery of active muscle properties following eccentric exercise: rabbit study with clinical relevance," British journal of sports medicine, vol. 47, no. 2, pp. 83-88, 2013.

[4] V. Davies, K. G. Thompson, and S.-M. Cooper, "The Effects Of Compression Garments On Recovery," (in English), Journal of Strength and Conditioning Research, vol. 23, no. 6, pp. 1786-94, Sep 2009

[5] J. R. Jakeman, C. Byrne, and R. G. Eston, "Lower limb compression garment improves recovery from exercise-induced muscle damage in young, active females," European Journal of Applied Physiology, vol. 109, no. 6, pp. 1137-1144, 2010.

[6] Y. Wang, P. Zhang, and Y. Zhang, "Experimental investigation the dynamic pressure attenuation of elastic fabric for compression garment," Textile Research Journal, vol. 84, no. 6, pp. $572-582,2014$

[7] C. L. Pruscino, S. Halson, and M. Hargreaves, "Effects of compression garments on recovery following intermittent exercise," European journal of applied physiology, vol. 113, no. 6, pp. 1585-1596, 2013.

[8] D. Marqués-Jiménez, J. Calleja-González, I. Arratibel, A. Delextrat, and N. Terrados, "Are compression garments effective for the recovery of exercise-induced muscle damage? A systematic review with meta-analysis," Physiology \& behavior, vol. 153, pp. 133-148, 2016.

[9] M. I. Trenell, K. B. Rooney, C. M. Sue, and C. H. Thomspon, "Compression garments and recovery from eccentric exercise: a 31P-MRS Study," Journal of sports science \& medicine, vol. 5, no. 1, p. 106, 2006.

[10] R. Duffield, J. Cannon, and M. King, "The effects of compression garments on recovery of muscle performance following high-intensity sprint and plyometric exercise," Journal of Science and Medicine in Sport, vol. 13, no. 1, pp. 136-140, 2010.

[11] M. E. Willems and E. C. Webb, "Effects of wearing graduated compression garment during eccentric exercise," Medicina Sportiva, vol. 14, no. 4, pp. 193-198, 2010.

[12] J. M. Fam and C. K. Ching, "Review on non-invasive risk stratification of sudden cardiac death," Proceedings of Singapore Healthcare, vol. 20, no. 4, pp. 263-278, 2011.

[13] R. Lamberts, J. Swart, B. Capostagno, T. Noakes, and M. Lambert, "Heart rate recovery as a guide to monitor fatigue and predict changes in performance parameters," Scandinavian journal of medicine \& science in sports, vol. 20, no. 3, pp. 449457, 2010.

[14] U. Dimkpa, "Post-exercise heart rate recovery: an index of cardiovascular fitness," J Exe Physiol, vol. 12, pp. 19-22, 2009.

[15] S. Y. Jae et al., "Slow heart rate recovery after exercise is associated with carotid atherosclerosis," Atherosclerosis, vol. 196, no. 1, pp. 256-261, 2008.

[16] T. Inoue, K. Iseki, C. Iseki, K. Kinjo, Y. Ohya, and S. Takishita, "Higher heart rate predicts the risk of developing hypertension in a normotensive screened cohort," Circulation Journal, vol. 71, no. 11, pp. 1755-1760, 2007.

[17] P. Cipresso et al., "Real Emotions for Simulated Social Networks," in Computational Social Networks: Springer, 2012, pp. 391-415.

[18] P. Kaikkonen, H. Rusko, and K. Martinmäki, "Post-exercise heart rate variability of endurance athletes after different highintensity exercise interventions," Scandinavian journal of medicine \& science in sports, vol. 18, no. 4, pp. 511-519, 2008.

[19] M. Felix and A. G. Dimitriu, "Orginal study the effects of physical training over QT dispersion and QTc dispersion to the beginner sportsmen," ed: Fascicula, 2011.

[20] M. C. Mandyam, E. Z. Soliman, A. Alonso, S. R. Heckbert, E. Vittinghoff, and G. M. Marcus, "A Longer Qt Interval is Associated with Increased Risk of Atrial Fibrillation," Circulation, vol. 126, no. 21 Supplement, p. A16639, 2012.

[21] M. Benn, B. Nordestgaard, S. Nielsen, M. Noerskov, G. Jensen, and A. Tybjaerg-Hansen, "Short and long QTc interval, cardiovascular disease and mortality," European Heart Journal, vol. 34, no. suppl 1, p. P4957, 2013.

[22] B. Dascombe, P. Laursen, K. Nosaka, and T. Polglaze, "No effect of upper body compression garments in elite flat-water kayakers," European journal of sport science, vol. 13, no. 4, pp. 341-349, 2013.

[23] A. S. Leicht, M. J. Crowe, W. H. Sinclair, and R. M. Sealey, "Influence of compression garments on physiological and perceptual responses during intermittent anaerobic running and recovery," Integrative Exercise Physiology, 2010.

[24] K. M. de Glanville and M. J. Hamlin, "Positive effect of lower body compression garments on subsequent $40-\mathrm{kM}$ cycling time trial performance," The Journal of Strength \& Conditioning Research, vol. 26, no. 2, pp. 480-486, 2012.

[25] D. Rimaud, L. Messonnier, J. Castells, X. Devillard, and P Calmels, "Effects of compression stockings during exercise and recovery on blood lactate kinetics," European journal of applied physiology, vol. 110, no. 2, pp. 425-433, 2010.

[26] M. W. Driller and S. L. Halson, "The effects of wearing lower body compression garments during a cycling performance test," Int J Sports Physiol Perform, vol. 8, pp. 300-306, 2013.

[27] L. T. N. Nguyen, D. Eager, and H. T. Nguyen, "Effect Of Wearing Whole Body Compression Garments On Cardiovascular Function Using Ecg Signals," Age (year), vol. 26, no. 3.1, p. 24, 2016.

[28] L. T. N. Nguyen, D. Eager, and H. T. Nguyen, "Effect Of Wearing Whole Body Compression Garments On Cardiovascular Function Using Ecg Signals," Telehealth and Assistive Technology, vol. 26, no. 3.1, pp. 24-29, 2016.

[29] H. Yeom and H. C. Kim, "MPS 08-04 The association between ST interval and blood pressure in a general population of Korea," Journal of Hypertension, vol. 34, p. e261, 2016.

[30] S. E. Privett, K. P. George, G. P. Whyte, and N. T. Cable, "The effectiveness of compression garments and lower limb exercise on post-exercise blood pressure regulation in orthostatically intolerant athletes," Clinical Journal of Sport Medicine, vol. 20, no. 5, pp. 362-367, 2010. 